\title{
Improved Long-Term Memory via Enhancing cGMP-PKG Signaling Requires CAMP-PKA Signaling
}

\author{
Eva Bollen ${ }^{1,5,6}$, Daniela Puzzo ${ }^{2,6}$, Kris Rutten 1,7, Lucia Privitera ${ }^{2}$, Jochen De Vry', Tim Vanmierlo',8, \\ Gunter Kenis', Agostino Palmeri', Rudi D'Hooge ${ }^{3}$, Detlef Balschun ${ }^{3}$, Harry MW Steinbusch', \\ Arjan Blokland ${ }^{4}$ and Jos Prickaerts*, I \\ 'Department of Psychiatry and Neuropsychology, School for Mental Health and Neuroscience, Maastricht University, Maastricht, The \\ Netherlands; '2Department of Bio-Medical Sciences, Section of Physiology, University of Catania, Catania, Italy; ${ }^{3}$ Laboratory of Biological \\ Psychology, Faculty of Psychology and Educational Sciences, University of Leuven, Leuven, Belgium; ${ }^{4}$ Department of Neuropsychology and \\ Psychopharmacology, Maastricht University, Maastricht, The Netherlands
}

\begin{abstract}
Memory consolidation is defined by the stabilization of a memory trace after acquisition, and consists of numerous molecular cascades that mediate synaptic plasticity. Commonly, a distinction is made between an early and a late consolidation phase, in which early refers to the first hours in which labile synaptic changes occur, whereas late consolidation relates to stable and long-lasting synaptic changes induced by de novo protein synthesis. How these phases are linked at a molecular level is not yet clear. Here we studied the interaction of the cyclic nucleotide-mediated pathways during the different phases of memory consolidation in rodents. In addition, the same pathways were studied in a model of neuronal plasticity, long-term potentiation (LTP). We demonstrated that cGMP/protein kinase G (PKG) signaling mediates early memory consolidation as well as early-phase LTP, whereas CAMP/protein kinase A (PKA) signaling mediates late consolidation and late-phase-like LTP. In addition, we show for the first time that early-phase cGMP/PKG signaling requires late-phase cAMP/PKA-signaling in both LTP and long-term memory formation.

Neuropsychopharmacology (20I4) 39, 2497-2505; doi:I0.1038/npp.20I4.106; published online 25 June 2014
\end{abstract}

\section{INTRODUCTION}

Memory is a complex, multifaceted phenomenon, in which a differentiation is made between acquisition, consolidation, and retrieval processes. Each of these processes relies on specific molecular mechanisms (Izquierdo et al, 2006; Izquierdo and Medina, 1997). Consolidation is a temporally graded process, in which newly acquired information becomes stabilized and stored in memory traces (Kandel, 2001). The cellular substrates of memory consolidation are molecular transformations at the participating synapses. They are labile and protein synthesis independent during

\footnotetext{
*Correspondence: Dr J Prickaerts, Department of Psychiatry and Neuropsychology, School for Mental Health and Neuroscience, Maastricht University, PO Box 616, 6200 MD Maastricht, The Netherlands, Tel: + 3 I 43 388II68, Fax: + 3 I 43 3884086, E-mail: jos.prickaerts@ maastrichtuniversity.nl

${ }^{5}$ Current address: Department of Behavioral Neuroscience and Drug Development, Institute of Pharmacology, Polish Academy of Sciences, 31-343 Kraków, Poland

${ }^{6}$ These authors contributed equally to this work.

${ }^{7}$ Current address: Department of Pharmacology Pain, Global Biomedical Sciences, Grünenthal GmbH, 52078 Aachen, Germany

${ }^{8}$ Current address: Department of Immunology and Biochemistry, Biomedical Research Institute, Hasselt University, 3590 Diepenbeek, Belgium

Received 2I September 20 I3; revised 28 February 20।4; accepted 28 February 2014; accepted article preview online 12 May 2014
}

the first hours $(<3 \mathrm{~h})$ after learning, but implement longlasting structural modifications during later phases, which rely on de novo protein synthesis. It has become evident that cyclic nucleotides, ie, cyclic AMP (cAMP) and cyclic GMP (cGMP), have an important role in memory consolidation and in a specific neuroplasticity phenomenon, which is generally regarded as the neural correlate of memory, ie, long-term potentiation (LTP; Bach et al, 1999; Bernabeu et al, 1996; Bourtchouladze et al, 1998; Frey et al, 1993; Lu et al, 1999; Prickaerts et al, 2002a; Son et al, 1998). Furthermore, cyclic nucleotides have been suggested to be differentially involved in distinct phases of the memory consolidation process. That is, cGMP has been attributed a role in early consolidation, whereas cAMP is implicated in late consolidation processes (Izquierdo et al, 2006; Rutten et al, 2007b). However, how these cyclic nucleotide-mediated memory processes are linked to each other is not clear.

Phosphodiesterases (PDEs) are enzymes that hydrolyze cAMP and/or cGMP, in the body and the brain. It has been previously shown that different inhibitors of PDEs enhance memory formation in rodents in a wide array of memory tasks (Reneerkens et al, 2009). These studies led to the identification of a number of PDE subfamilies as promising targets for memory improvement, in specific the PDE1, PDE2, PDE4, PDE5, and PDE9 subtypes. In this study, we focused on cAMP-selective PDE4 (Barad et al, 1998; Rose et al, 2005; Rutten et al, 2007a; Zhang et al, 2005), cGMPselective PDE5 (Prickaerts et al, 2004; Rutten et al, 2005), 
and PDE2, which hydrolyzes both cAMP and cGMP (Boess et al, 2004; Rutten et al, 2007b). It has been suggested that the cognition-enhancing effects of PDE inhibitors are related to activation of $\mathrm{cAMP} /$ protein kinase $\mathrm{A}$ (PKA)/ cAMP responsive element-binding protein (CREB) and cGMP/protein kinase G (PKG)/CREB signaling pathways (Blokland et al, 2006; Reneerkens et al, 2009; Rutten et al, 2007b), which are both associated with late-phase LTP (L-LTP). In contrast to the transient early phase of LTP (E-LTP), this long-lasting form of LTP is dependent on protein synthesis via CREB phosphorylation (Barad et al, 1998; Frey et al, 1993; Lu et al, 1999; Son et al, 1998). In the present study, the exact role of hippocampal cyclic nucleotides in early $v s$ late phases of memory consolidation in the object recognition task (ORT) as well as in an early and late phase of LTP was investigated.

We hypothesized that early consolidation of object memory is dependent on CGMP-PKG signaling and that late consolidation is dependent on cAMP-PKA signaling in the hippocampus. To investigate these mechanisms and their relationship in an in vivo behavioral setup, we coadministered PDE inhibitors peripherally and protein kinase (PK) inhibitors intra-hippocampally. We assessed the effect of PDE inhibition on early and late consolidation processes in an ORT and whenever memory improvement was observed, we aimed to block this effect with centrally administered PKG and PKA inhibitors. In addition, the involvement of the two cyclic nucleotide-mediated pathways was evaluated in different phases of LTP in hippocampal slices in vitro. This study provides further evidence for differential time-dependent contribution of cAMP and cGMP in memory consolidation and synaptic plasticity, and it shows that for long-term memory improvement the cAMP-PKA pathway is required after cGMP signaling.

\section{MATERIALS AND METHODS}

\section{Subjects}

All experimental procedures were approved by the Local Ethical Committee for Animal Experiments of Maastricht University or of University of Catania, and were in agreement with the respective governmental guidelines. For behavioral experiments, 3- to 6-month-old male Wistar rats (Charles River, Sulzfeld, Germany) were used. For electrophysiological studies, C57BL/6J male mice, 3-month old, were obtained from a breeding colony housed in the animal facility of the University of Catania.

Rats were individually housed in standard type 3 Makrolon cages on sawdust bedding. The animals were held in an airconditioned room (approximately $21^{\circ} \mathrm{C}$ ) and had free access to food and water. A softly playing radio provided background noise. A reversed light-dark cycle was applied in the room (lights on between 0700 and 1900 hours) in order to test the animals during their naturally active period. Housing conditions of the mice were the same as for rats, except that they were housed socially with five animals per cage.

\section{Object Recognition}

Apparatus. Animals were subjected to the ORT. This task was performed in a circular arena with a diameter of $83 \mathrm{~cm}$ and walls of $40 \mathrm{~cm}$ high. The backside half of the arena wall was made of gray polyvinyl chloride, and the front half of transparent polyvinyl chloride. The objects consisted of four sets including (1) a cone made of brass, (2) a transparent glass bottle, (3) a massive metal beam with two holes and (4) a massive aluminum cube with a tapered top. The animals were unable to displace the objects. All objects were present in threefold and were cleaned thoroughly after each trial to remove all olfactory traces.

Procedure. During the first weeks of the experiment, the animals were habituated to handling and observation by the experimenter. In addition, animals were familiarized to the environment and procedures.

ORT procedures were adapted from previous studies (Ennaceur and Delacour, 1988), with modifications as described previously (Prickaerts et al, 1997). During the ORT, rats were put in a circular arena, facing the middle of the transparent wall. In the arena, two identical objects were placed approximately $10 \mathrm{~cm}$ from the wall. The animal was given 3 min to explore the environment and objects. The time spent exploring the separate objects was manually scored using a personal computer by an experimenter who was blinded to the conditions tested. Exploration was defined as directing the nose to the object, with a maximal distance between nose and object of $2 \mathrm{~cm}$. Leaning or sitting on the object was not considered exploratory behavior. After $24 \mathrm{~h}$, the rat was put back in the arena. In this second trial, one of the objects from the initial trial was replaced by another object. Again the rat was allowed to explore the objects for $3 \mathrm{~min}$ and object exploration times were recorded. An increase in time spent exploring the new object was interpreted as recognition of the previously encountered objects. A relative measure of discrimination between the old and new objects was calculated, which was corrected for total exploration time. The resulting discrimination index (exploration time new object-exploration time old object)/(exploration time new object + exploration time old object) reflects recognition memory independent of normal exploratory behavior (Akkerman et al, 2012). Animals that did not show normal exploration $(<7 \mathrm{~s})$ were excluded from analysis.

The test regime included two 24-h interval testing sessions a week. We opted for a delay interval of $24 \mathrm{~h}$, at which under normal, non-treated circumstances, no discrimination between the objects occurs, which allows for an improvement of long-term memory performance following drug treatment. Testing was done between 0900 and 1700 hours under red light conditions.

\section{Drug Administration}

Cannula placement. To inject into the CA1 of the hippocampus, cannulae were implanted by means of stereotaxic surgery. Animals were fixed in a stereotaxic frame after induction of full anesthesia with isoflurane (induction: 5\%; maintenance: $2 \%$ ). Cannulae were placed bilaterally in hand drilled holes above the CA1 region of the hippocampus at following coordinates: $-3.6 \mathrm{~mm}$ anterior, $\pm 3.0 \mathrm{~mm}$ lateral and $3.0 \mathrm{~mm}$ ventral from bregma (Paxinos and Watson, 1998). When cannulae were in place they were fixed to the skull using acrylic dental cement (Paladur) and small screws. Animals were allowed to recover from surgery for 
2 weeks before the testing procedures started. In order to verify correct cannula location, the central infusion procedure was repeated at the end of the experiment injecting $1 \mu \mathrm{l}$ $5 \%$ methylene blue/ $95 \%$ saline bilaterally. Ten minutes after the methylene blue injections, animals were decapitated and their brains were rapidly removed. The correct injection location was verified by evaluating the methylene blue discoloration of the hippocampus.

Treatments. We administered three selective PDE inhibitors: PDE2 inhibitor BAY 60-7550 ( $\mathrm{IC}_{50}: 4.7 \mathrm{nM}$; kindly donated by BAYER AG, Wuppertal, Germany), PDE4 inhibitor rolipram $\left(\mathrm{IC}_{50}: 1.5 \mathrm{nM}\right.$; Sigma Aldrich, Zwijndrecht, the Netherlands) and PDE5 inhibitor vardenafil $\left(\mathrm{IC}_{50}: 0.7 \mathrm{nM}\right.$; kindly donated by BAYER AG). All three PDE inhibitors have been shown to cross the blood-brain barrier (Krause and Kuhne, 1988; Reneerkens et al, 2012; Reneerkens et al, 2013). The PKA inhibitor Rp-8-Br-cAMPS and PKG inhibitor Rp-8-Br-cGMPS were obtained from Biolog (Bremen, Germany). First, rats were treated solely with the PDE inhibitors (combined with central vehicle (saline) infusion) on both time points. Whenever a PDE inhibitor significantly improved memory performance, we subsequently attempted to block this effect using co-infusion of a PKG or PKA inhibitor. Drugs were administrated immediately (ie, 4-10 min after the start of the learning trial) or $3 \mathrm{~h}$ after the first trial. This resulted in a total of 17 treatment combinations of different PDE inhibitors (vehicle, PDE2, PDE4, or PDE5 inhibition) with the PK inhibitors (vehicle, PKA, or PKG inhibitor) on the different time points of administration $(\mathrm{T} 1+0 \mathrm{~h}$ or $\mathrm{T} 1+3 \mathrm{~h})$. All solutions were freshly dissolved on the day of testing. Peripheral drug administration of the PDE-I was done either orally (BAY 60$75503 \mathrm{mg} / \mathrm{kg}$ and vardenafil $1 \mathrm{mg} / \mathrm{kg}$ ) or intraperitoneally (rolipram $0.03 \mathrm{mg} / \mathrm{kg}$ ). All PDE inhibitors were dissolved in the same vehicle ( $98 \%$ methyl cellulose (tylose) solution $(0.5 \%)$ and $2 \%$ tween 80$)$ and administered in a volume of $2 \mathrm{ml} / \mathrm{kg}$. Inhibitors of PKs were administered directly into the hippocampus via the surgically implanted guiding cannulae. Using a micropump, $0.5 \mu$ l saline $(0.9 \% \mathrm{NaCl})$ or a saline solution containing RP-8-Br-cAMPS or RP-8-BrcGMPS (both $2 \mu \mathrm{g} / \mu \mathrm{l}$ ) was injected bilaterally over a time period of 1 min through the infusion cannulae, which were connected to two $10 \mu$ l Hamilton syringes by polyethylene tubes. The injection needles were left in place for an additional minute to prevent reflux of infused drugs along the cannula track. Drug dosages and administration routes were based on previous studies (Boess et al, 2004; Prickaerts et al, 2002b; Rutten et al, 2006).

For electrophysiological experiments, vardenafil $(10 \mathrm{nM})$, rolipram (100 nM), Rp-8-Br-cGMPS $(10 \mu \mathrm{M})$, and Rp-8-BrcAMPS $(20 \mu \mathrm{M})$ were diluted in artificial CSF (ACSF) immediately before use, and applied in the bath solution at different time points before or after the induction of LTP. The concentrations were based on previous literature (Barad et al, 1998; Puzzo et al, 2009).

\section{Electrophysiology}

Electrophysiological recordings were performed as previously described (Puzzo et al, 2009). Briefly, transverse hippocampal slices $(400 \mu \mathrm{m})$ were cut and transferred to a recording chamber where they were maintained at $29^{\circ} \mathrm{C}$ and perfused with ACSF (flow rate $2 \mathrm{ml} / \mathrm{min}$ ) continuously bubbled with $95 \% \mathrm{O}_{2}$ and $5 \% \mathrm{CO}_{2}$. The ACSF composition was composed of the following (in $\mathrm{mM}$ ): $124.0 \mathrm{NaCl}, 4.4$ $\mathrm{KCl}, 1.0 \mathrm{Na}_{2} \mathrm{HPO}_{4}, 25.0 \mathrm{NaHCO}_{3}, 2.0 \mathrm{CaCl}_{2}, 2.0 \mathrm{MgSO}_{4}$, and 10.0 glucose. Field extracellular recordings were performed by stimulating the Schaeffer collateral fibers through a bipolar tungsten electrode and recording in CA1 stratum radiatum with a glass electrode filled with ACSF. A $15 \mathrm{~min}$ baseline was recorded every minute at an intensity that evoked a response approximately $35 \%$ of the maximum evoked response. Early LTP was induced by a weak tetanus (4 pulses at $100 \mathrm{~Hz}$, with the bursts repeated at $5 \mathrm{~Hz}$ and one tetanus of 10-burst trains; Chapman et al, 1999; Puzzo et al, 2009; Zakharenko et al, 2003). Responses were recorded for $3 \mathrm{~h}$ after tetanization and measured as field excitatory post-synaptic potentials slope expressed as percentage of baseline. The results were expressed as mean \pm standard error mean.

\section{Statistical Analysis}

A one-way ANOVA was performed to investigate effects of treatment on discrimination index for every type of PDE inhibitor separately. In case of significant effects between group, we used Fischer's LSD for post hoc comparisons. For LTP, statistical analysis was performed with two-way ANOVA with repeated measures. For all analyses, significance level was set at 0.05 .

\section{RESULTS}

\section{Object Memory}

Effect of cGMP-selective PDE5 inhibition is PKG dependent and limited to early consolidation. Memory performance was assessed in the ORT with a 24-h interval in rats. Differences were found in discrimination performance for the different PDE5 inhibition conditions $(\mathrm{F}(4,93)=3.18$; $p<0.05$ ), which are summarized in Figure 1a. When compared with vehicle condition, vardenafil in combination with an intra-hippocampal saline injection enhanced memory when given immediately $(\mathrm{T}+0 \mathrm{~h})$ after the first trial $(p<0.05)$, but not when administered $3 \mathrm{~h}$ after the first trial (n.s.). Memory improvement after immediate vardenafil treatment remained when the administration of the PDE5 inhibitor was combined with an intra-hippocampal injection of PKA inhibitor (n.s.). However, when vardenafil was co-administered with PKG inhibition directly after learning, the memory enhancement tended to be reduced $(p=0.056)$.

Effect of cAMP-selective PDE4 inhibition is PKA dependent and limited to late consolidation. Differences were found on discrimination performance for the different PDE4 inhibition conditions $(F(4,94)=2.74 ; p<0.05)$, which are summarized in Figure 1b. Rolipram improved memory when it was injected $3 \mathrm{~h}$ after the first trial, in combination with intra-hippocampal saline infusions $(p<0.05)$. However, this improvement was not present when drugs were administered immediately after learning (n.s.). The memory enhancement after delayed rolipram administration $(\mathrm{T}+3 \mathrm{~h})$ was unaffected when RP-8-Br-cGMPS was 

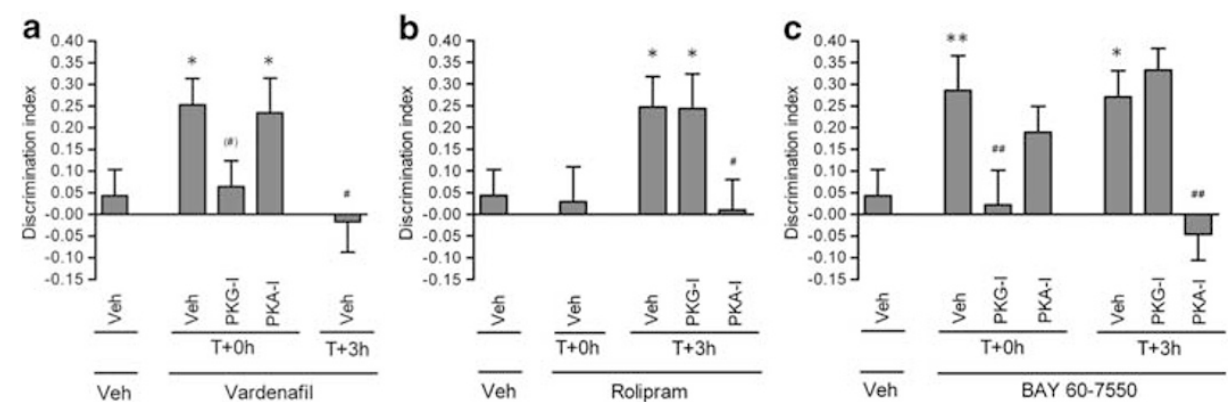

Figure I The effects of PDE inhibition treatment. (a) PDE5 inhibition-Vardenafil I mg/kg, p.o., $n=26 / 18 / / 8 / / 8 / 18$; (b) PDE4 inhibition—Rolipram $0.03 \mathrm{mg} / \mathrm{kg}$, i.p., $n=26 / 18 / 18 / 19 / 18$; (c) PDE2 inhibition-BAY 60-7550 $3 \mathrm{mg} / \mathrm{kg}, \quad$ p.o., $n=26 / 19 / 18 / 19 / 18 / 16 / 19$ on discrimination performance (discrimination index; means + SEM) in a 24-h-delay object recognition task. PDE inhibition was administered immediately after $(T+0 \mathrm{~h})$ or $3 \mathrm{~h}(T+3 \mathrm{~h})$ after the first trial. When PDE inhibition combined with saline intra-hippocampal injections (veh) yielded significant improvement of discrimination, we subsequently combined PDE treatment with inhibitors of PKG (PKG-l; RP-8-Br-cGMPS I $\mu g / s i d e)$ or PKA (PKA-l; RP-8-Br-cAMPS I $\mu$ g/side). Asterisks indicate significant differences $(* p<0.05$, $* * p<0.01$ ) of PDE inhibition treatment from vehicle condition. Hashes indicate a significant reversal of PDE-induced memory improvement $\left({ }^{(\#)} p=0.056,{ }^{\#} p<0.05,{ }^{\# \#} p<0.01\right)$.

intra-hippocampally co-administered (n.s.), whereas RP-8Br-cAMPS blocked the improvement $(p<0.05)$.

Effect of PDE2 inhibition involves both early and late consolidation and is PKG- and PKA-dependent. Differences were found on discrimination performance for the different PDE2 inhibition conditions $(\mathrm{F}(6,128)=4.53$; $p<0.001$ ), which are summarized in Figure 1c. In comparison with the vehicle condition, BAY 60-7550 enhanced memory after both immediate $(p<0.01)$ and delayed administration $(p<0.05)$. The immediate improvement was fully prevented by co-administration of a PKG inhibitor $(p<0.01)$, but remained when PDE2 inhibition was combined with a PKA inhibitor (n.s.). When memory enhancement was induced by delayed injections of BAY 607550 , these effects were blocked by intra-hippocampal infusions of RP-8-Br-cAMPS $(p<0.01)$. In this case, however, co-administration of RP-8-Br-cGMPS could not block memory enhancement (n.s.).

Memory enhancing effects of cGMP-targeting PDEs are dependent on subsequent PKA activity. To investigate whether memory improvement after immediate treatment with cGMP-targeting PDE inhibitors relies on cAMP-PKA signaling during late consolidation, immediate vardenafil and BAY 60-7550 administration were combined with PKA inhibition $3 \mathrm{~h}$ after learning. Results are summarized in Figure 2. For vardenafil treatment, significant changes in discrimination performance were found $(\mathrm{F}(2,38)=5.62$; $p<0.01$; Figure $2 a$ ) with animals that received only vardenafil performing better than the vehicle group $(p<0.05)$, and a reversal to vehicle levels in combined treatment with vardenafil and Rp-8-Br-cAMPS $(p<0.01)$. For BAY $60-7550$ treatment we found similar changes in memory performance $(\mathrm{F}(2,36)=3,97 ; p<0.05$; Figure $2 \mathrm{~b})$. That is, BAY 60-7550 enhanced discrimination $(p<0.05)$, whereas combining BAY 60-7550 with delayed PKA inhibition reversed the improvement $(p<0.05)$.

Delayed cAMP stimulation enhances memory performance, regardless of preceding blockage of cGMP signaling. We evaluated the dependency of cAMP-mediated memory enhancement on preceding cGMP activity, by combining immediate intrahippocampal administration of $\mathrm{Rp}-8$-Br-cGMPS with rolipram injected $3 \mathrm{~h}$ after learning (Figure 3a). This resulted in significant changes in discrimination performance $(\mathrm{F}(2,33)=3.38 ; p<0.05)$. Memory improving effects of rolipram could not be precluded with immediate Rp-8-Br-cGMPS injections $(p<0.05)$. In addition, we replicated these findings for memory enhancing effects after administration of BAY 60-7550 $(\mathrm{F}(2,27)=3.63$; $p<0.05$; Figure $3 b$ ). Again, Rp-8-Br-cGMPS could not prevent improved discrimination performance caused by delayed BAY 60-7550 treatment $(p<0.05)$.

Effects of altered cyclic nucleotide signaling on LTP. Electrophysiological experiments were performed by using a weak tetanus to produce E-LTP, ie, a transient potentiation, at Schaffer collateral-CA1 synapses in hippocampal slices. Similar to behavioral studies, the PDE5 inhibitor vardenafil $(10 \mathrm{nM})$ produced a longer-lasting potentiation when administered before $(\mathrm{F}(1,14)=10.53, \quad p<0.01$; Figure 4a), but not when administered 90 min after tetanus $(\mathrm{F}(1,13)=0.874$; n.s. $)$. The effect of vardenafil administration $10 \mathrm{~min}$ after LTP induction did not differ from potentiation after vardenafil administration $10 \mathrm{~min}$ before induction $(\mathrm{F}(1,13)=0.210$; n.s.; Figure $4 \mathrm{~d})$. No effects on baseline transmission were observed (Supplementary Figure 1). The effect of vardenafil was blocked by coperfusion with the PKG inhibitor Rp-8-Br-cGMPS $(10 \mu \mathrm{M}$; $\mathrm{F}(1,14)=0.01$; n.s. compared with vehicle; Figure $4 \mathrm{a})$. The positive effect of vardenafil on LTP was also blocked by the PKA inhibitor Rp-8-Br-cAMPS $(20 \mu \mathrm{M}$ for $30 \mathrm{~min}$; $\mathrm{F}(1,13)=$ 0.04 , n.s.; $\mathrm{F}(1,13)=8.86, p<0.05$; comparing vardenafil and vardenafil + Rp-8-Br-cAMPS before and after Rp-8-BrcAMPS administration, respectively; Figure $4 \mathrm{~b}$ ), but only when administered $90 \mathrm{~min}$ after tetanus and not when administered together at time of induction $(\mathrm{F}(1,13)=0.061$; n.s.; Figure 4c). The time window at $90 \mathrm{~min}$ after induction was identified by applying $\mathrm{Rp}-8$-Br-cAMPS at various time points after vardenafil facilitated LTP induction (Supplementary Figure $2 ; \mathrm{t}(8)=39.41, p<0.0001$ compared with vardenafil-treated slices).

We then tested whether inhibition of PDE4 affected LTP. Slices treated with the PDE4 inhibitor rolipram (100 nM) 

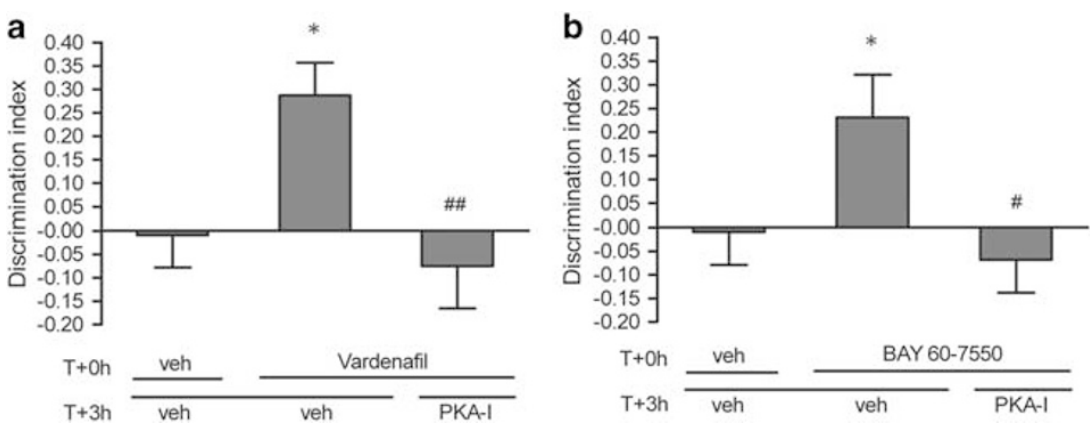

Figure 2 The effects of early cGMP-targeting PDE inhibition treatment. (a) PDE5 inhibition-Vardenafil I mg/kg, n=28//3/l4; (b) PDE2 inhibition—BAY 60-7550 $3 \mathrm{mg} / \mathrm{kg}, n=28 / / 2 / 13$ ) in combination with PKA inhibition (PKA-l; RP-8-Br-cAMPS; i.h.; I $\mu \mathrm{g} / \mathrm{side}$ ) $3 \mathrm{~h}$ (T $+3 \mathrm{~h}$ ) after the first trial on discrimination performance (discrimination index; means + SEM) in a 24-h-delay object recognition task. Asterisks indicate significant differences $(* p<0.05)$ of PDE inhibition treatment from vehicle condition. Hashes indicate a significant reversal of PDE-induced memory improvement $\left({ }^{\#} p<0.05,{ }^{\# \#} p<0.0 \mathrm{I}\right)$.
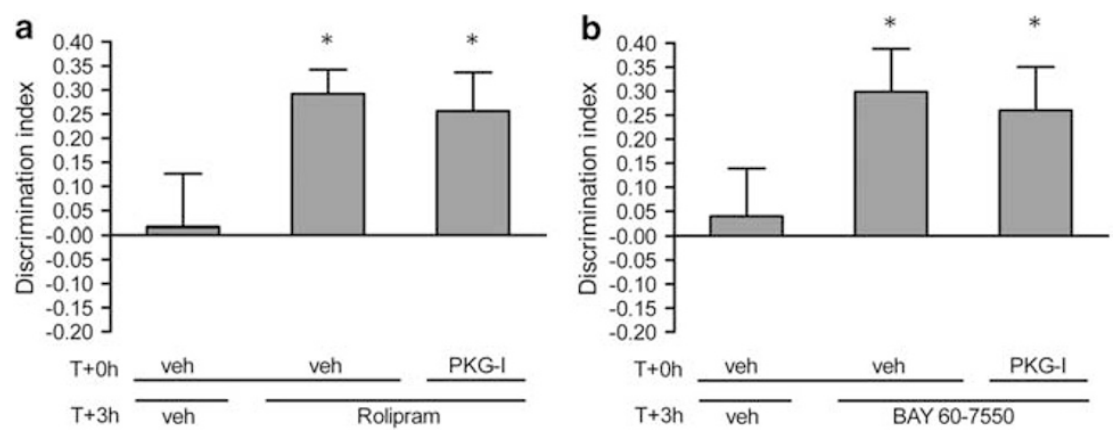

Figure 3 The effects of cAMP-targeting PDE inhibition treatment. (a) PDE4 inhibition—Rolipram 0.03 mg/kg, $n=10 / / 4 / I 2 ;$ (b) PDE2 inhibition—BAY 60-7550 $3 \mathrm{mg} / \mathrm{kg}, n=9 / 10 / \mathrm{I}$ ) administered $3 \mathrm{~h}$ after learning in combination with PKG inhibition (PKG-l; RP-8-Br-cGMPS; i.h.; I $\mu$ g/side) immediately after the first trial on discrimination performance (discrimination index; means + SEM) in a 24-h-delay object recognition task. Asterisks indicate significant differences $(* p<0.05)$ of PDE inhibition treatment from vehicle condition.

90 min after a weak tetanus showed an enhancement of LTP $(\mathrm{F}(1,12)=12.91 ; p<0.01$ compared with vehicle; Figure $5 \mathrm{a})$. Bath application of rolipram before tetanus yielded in an enhanced LTP $(\mathrm{F}(1,12)=5.659 ; p<0.05)$, whereas $10 \mathrm{~min}$ after tetanus rolipram did not affect $\operatorname{LTP}(\mathrm{F}(1,13)=0.001$; n.s.; Figure 5d). The facilitation observed after rolipram administration at 90 min after tetanus could be blocked by co-perfusion with Rp-8-Br-cAMPS. This blockage was partial as LTP was still enhanced compared with vehicle, which might be a matter of dosing of rolipram, ie, too high, and/or Rp-8-Br-cAMPS, ie, too low. Importantly, the effect of rolipram was specific to LTP because it did not affect basal synaptic transmission $(\mathrm{F}(2,12)=2.49$, n.s.; Supplementary Figure 1). When Rp-8-Br-cGMPS $(10 \mu \mathrm{M})$ was applied alone before tetanus it led to a siginificant decrease of E-LTP when compared with vehicle-treated slices $(\mathrm{F}(1,14)=12.36 ; p<0.01)$. Importantly, however, the rolipram-induced improvement of LTP was not significantly changed $(\mathrm{F}(1,10)=1.43$; n.s.; Figure $5 \mathrm{~b})$. Application of Rp8-Br-cGMPS together with rolipram at $90 \mathrm{~min}$ after induction resulted in a similar level of enhanced LTP as with rolipram application alone $(\mathrm{F}(1,11)=0.317$; n.s.; Figure $5 \mathrm{c})$.

\section{DISCUSSION}

The present study replicated our previous findings (Rutten et al, 2007b) by showing that inhibition of PDE2, 4, and
5 can prolong retention in an ORT, although these PDE subtypes act differentially on early and late stages of memory consolidation depending on their target cyclic nucleotide. That is, inhibition of PDE5 (by vardenafil), a cGMP-targeting PDE subtype, during early stages of consolidation and synaptic plasticity improved memory formation and as was now also shown LTP, ie, it converted a E-LTP into a longer-lasting L-LTP-like signal. A cAMPspecific PDE4 inhibitor (by rolipram) showed a memory improving effect as well as the ability, as shown for the first time, to induce a longer-lasting LTP when applied during late consolidation and a later stage of synaptic plasticity, respectively. The fact that rolipram enhances synaptic plasticity when administered before, but not after LTP induction is line with previous literature (Barad et al, 1998), and might be explained by effects only on acquisition-like processes, which we also found to be independent of PKA signaling. Finally, inhibition of PDE2 (by BAY 60-7550), which regulates levels of both cAMP and cGMP, effectively enhanced memory performance when administered directly as well as $3 \mathrm{~h}$ after learning.

In addition, we demonstrated that it is possible to counteract the memory-enhancing effects of PDE inhibitors by intra-hippocampal co-administration of an inhibitor of PKA or PKG, two important effector proteins of cAMP and cGMP, respectively. Specifically, memory enhancement as observed after early treatment with PDE5 or PDE2 inhibitors was successfully blocked only by co-administra- 

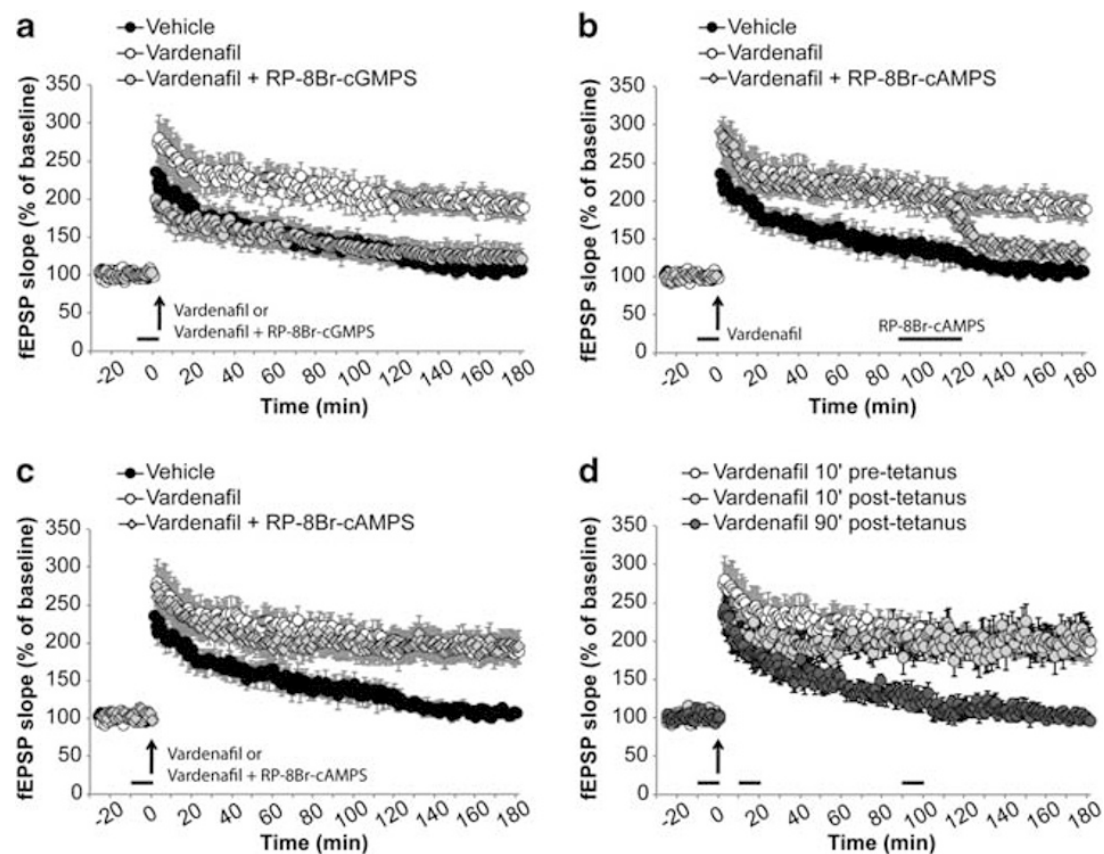

Figure 4 Effects of vardenafil and PKG/PKA inhibitors on hippocampal LTP. (a) Ten minutes perfusion of hippocampal slices with vardenafil (I $0 \mathrm{nM}$ ) before tetanus increases levels of potentiation when compared with vehicle-treated slices $(n=8 / 8)$; PKG inhibition by Rp-8-Br-cGMPS (I $0 \mu M)$ reverses the vardenafil-induced LTP improvement $(n=8)$. (b) The increase of LTP induced by vardenafil before tetanus is blocked by a 30-min perfusion with $\mathrm{Rp}$-8-BrcAMPS $(20 \mu M)$ administered at 90 min after tetanus $(n=7$; comparing vardenafil and vardenafil + Rp-8-Br-cAMPS before and after Rp-8-Br-cAMPS administration). (c) Perfusion with Rp-8-Br-cAMPS concomitant to vardenafil 10 min before tetanus does not affect the vardenafil-induced potentiation ( $n=7$; comparing vardenafil and vardenafil + Rp-8-Br-cAMPS before tetanus). (d) Vardenafil increases potentiation when administered I0 min before or after tetanus ( $n=7$; comparing vardenafil before and after tetanus), whereas it does not have any effect at 90 min after tetanus ( $n=7$; comparing vardenafil and vehicle). Arrow indicates tetanus delivery (one 10-burst stimulation-weak tetanus) and horizontal bars indicate the period during which drugs were added to the bath solution.

tion of the PKG inhibitor RP-8-Br-cGMPS, whereas memory improvement remained intact when combined with the PKA inhibitor RP-8-Br-cAMPS. PDE4 or PDE2 inhibitors improved memory when administered during late consolidation processes and co-administration of a PKA inhibitor prevented this enhancement. However, co-administration of a PKG inhibitor did not hinder the memory improvement induced by PDE inhibitors that target cAMP. These findings were again replicated for PDE4 and PDE5 inhibition in in vitro LTP measurements in hippocampal slices. This has major implications for treatment with cognition enhancing drugs, which improve specifically the cGMP and/or cAMP signaling cascades. That is, timing of treatment is essential to optimally influence memory consolidation processes after learning.

The results of this study show that the memory-enhancing effects of cGMP- and cAMP-selective PDE inhibitors are mediated by cGMP-PKG and cAMP-PKA signaling, respectively. Only a few studies have attempted to provide direct evidence for the contribution of these cascades in the behavioral effects induced by PDE inhibition. Devan et al (2007) blocked cGMP-PKG signaling in vivo through upstream inhibition of nitric oxide synthase, and could attenuate subsequent memory impairment with a PDE5 inhibitor. In accordance with our present findings, Kroker et al (2012) were able to convert E-LTP into L-LTP by increasing cGMP via a PDE9 inhibitor, which was blocked by co-application with a PKG inhibitor. Moreover, our results now show that for cAMP as well as cGMP stimulation, the subsequent activation of their respective PKs is required for the memory-enhancing effects of PDE inhibition.

Improved memory formation because of enhancement of cGMP- and cAMP-signaling cascades is most likely attained through de novo protein synthesis caused by postsynaptic CREB-mediated transcription, although we do not provide direct evidence for this notion in this study. The critical role for CREB phosphorylation downstream of CGMP-PKG and cAMP-PKA signaling has been described in LTP studies (Lu and Hawkins, 2002; Navakkode et al, 2004; Taqatqeh et al, 2009). Furthermore, in the formation of several types of long-term memory, among which object recognition, the necessity of CRE gene-driven protein synthesis paradigms has been extensively reported. Previous studies demonstrated increased hippocampal levels of phosphorylated CREB after in vivo subchronic rolipram treatment (Monti et al, 2006), as well as after in vitro bath application of sildenafil on tetanized hippocampal slices in APP/PS1 mice (Puzzo et al, 2009) and aged mice (Palmeri et al, 2013). Although the transcriptional program of CREB phosphorylation is still largely unclear, it is known that the target genes of CREB are functionally heterogeneous, including channel subunits, other transcription factors, and growth factors (Sakamoto et al, 2011).

It has to be noted that vasodilation is one of the most evident effects of PDE inhibitors (Paterno et al, 1996). The memory-enhancing effects of PDE inhibition could therefore also be attributed to an increased central blood flow and glucose metabolism. However, in a previous study, we 

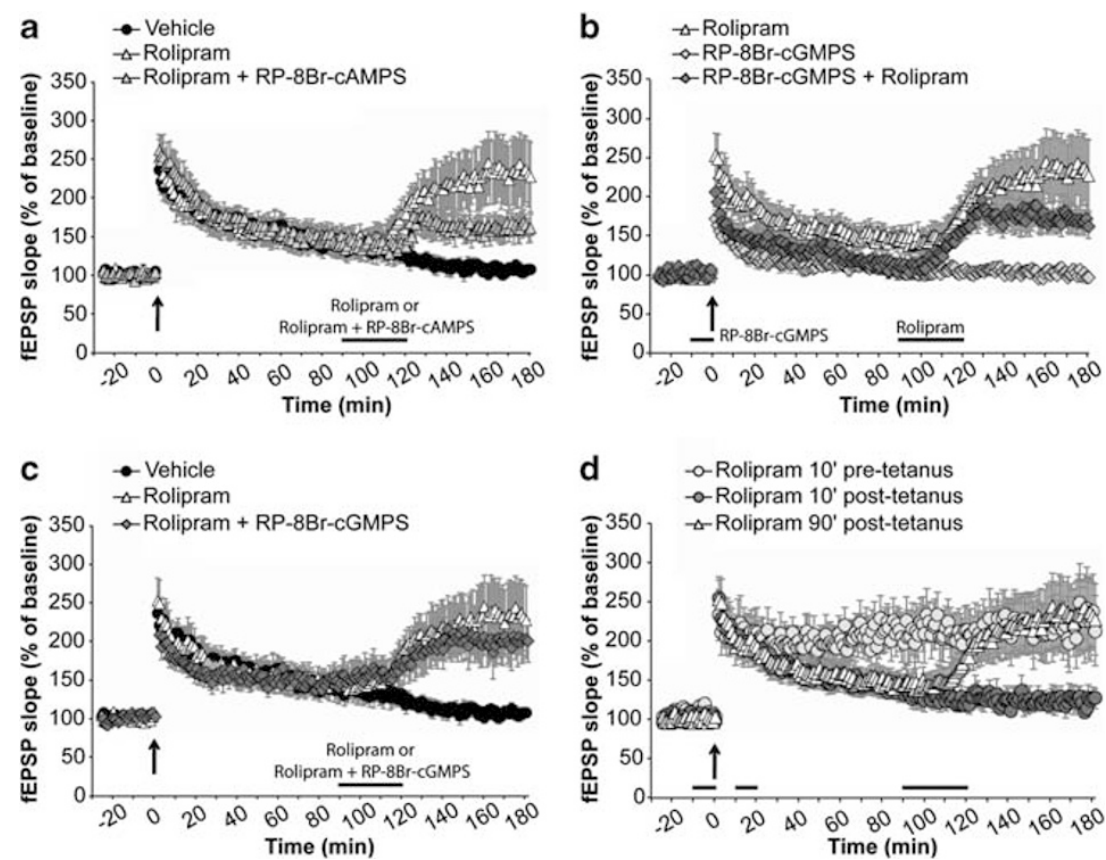

Figure 5 Effects of rolipram and PKG/PKA inhibitors on hippocampal LTP. (a) Perfusing hippocampal slices with rolipram (I $00 \mathrm{nM}$ ) 90 min after a weak tetanus boosts LTP in comparison to vehicle $(n=6 / 8)$. Co-perfusion with Rp-8-Br-cAMPS $(20 \mu \mathrm{M})$ induced a reduction of the effect of rolipram that, however, still shows an increased potentiation compared with vehicle $(n=5 / 8)$. (b) Brief perfusion with Rp-8-Br-cGMPS (I0 $\mu$ M) does not significantly decrease rolipram-induced improvement of LTP $(n=6 / 6)$. Yet Rp-8-Br-cGMPS alone decreases LTP (early-phase) compared with vehicle-treated slices $(n=8 / 8$; the latter conditions are shown in panel A). (c) Treatment with Rp-8-Br-cGMPS concomitant with rolipram 90 min after tetanus does not affect rolipram-induced LTP $(n=7$; comparing rolipram and rolipram + Rp-8-Br-cGMPS). (d) Rolipram increases potentiation when administered 10 min before tetanus $(n=7$; comparing rolipram with vehicle), whereas it does not have any effect at 10 min after tetanus $(n=7$; comparing rolipram with vehicle). Arrow indicates tetanus delivery (one $\theta$-burst stimulation — weak tetanus) and horizontal bars indicate the period during which drugs were added to the bath solution.

have demonstrated that the beneficial effects of systemically applied PDE inhibitors on memory in rats are independent of cerebrovascular effects (Rutten et al, 2009). In addition, in the present study, synaptic plasticity in hippocampal slices was enhanced after in vitro bath application of vardenafil and rolipram. Because of this, it is rather implausible that changes in blood flow contribute significantly to memory enhancement after PDE inhibition, but is more likely attributable to alterations in synaptic plasticity in the hippocampus.

This is further supported by the fact that when upregulating cyclic nucleotides, and thus activating their respective pathways, timing is of the essence: only immediate elevation of cGMP levels or increased cAMP levels $3 \mathrm{~h}$ after learning resulted in prolonged memory. This suggests that at times, despite the elevated levels of cyclic nucleotides, synaptic plasticity is not enhanced. Moreover, we demonstrated that the memory improvement caused by administration of cGMP-targeting PDE inhibitors immediately after learning can be blocked by infusion of a PKA inhibitor $3 \mathrm{~h}$ after learning. This important finding shows that for memory improvement the enhancement of cGMP-PKG signaling in early consolidation phases requires PKA signaling in a later stage of consolidation. cAMP-PKA signaling on the other hand is not reliant on previous cGMP activity during consolidation. This seemingly contradicts earlier LTP work that suggested that cAMP-PKA and cGMP-PKG act in parallel to activate CREB (Lu and Hawkins, 2002). However, in these studies, a difference in time windows was not investigated, which explains the apparent discrepancy. A similar sequential relationship between cGMP and cAMP during long-term memory formation has been suggested based on work in crickets by Matsumoto et al (2006). Strikingly, our LTP data again support our behavioral data as we observed that even though early PKG inhibition decreased E-LTP below vehicle levels, adding rolipram 90 min after induction still brought about enhanced synaptic plasticity, irrespective of the initial PKG inhibition. Thus, cAMP-PKA signaling acts independently of cGMP signaling to improve memory formation.

Our data indicate that cGMP and PKG may facilitate memory formation through mechanisms, which eventually lead to reinforcement of postsynaptic cAMP-PKA-CREB signaling. cGMP-PKG signaling has been shown to act as an intrinsic modulatory system, which regulates $\mathrm{Ca}^{2+}$ levels in the neuron, eg, through activation of cyclic nucleotide-gated channels (Matsumoto et al, 2006) or via the release of $\mathrm{Ca}^{2+}$ from ryanodine stores ( $\mathrm{Lu}$ and Hawkins, 2002). As such, elevated levels of cGMP and PKG can lower the stimulation threshold for $\mathrm{Ca}^{2+}$ signals from other sources, and therefore could enhance postsynaptic cAMP signaling. Our study implies that long-term memory improvement due to cGMPtargeting PDE inhibitors should be regarded as the result of cGMP-mediated modulation of cAMP pathways. The necessity of late consolidation processes for long-term memory consolidation corresponds to the notion that during the early phase of memory consolidation synaptic changes are labile and require stabilization later on in the memory formation process (Kandel, 2001). See Figure 6 for a hypothetical scheme illustrating how postsynaptic cGMP and cAMP signaling could be involved in memory formation. 


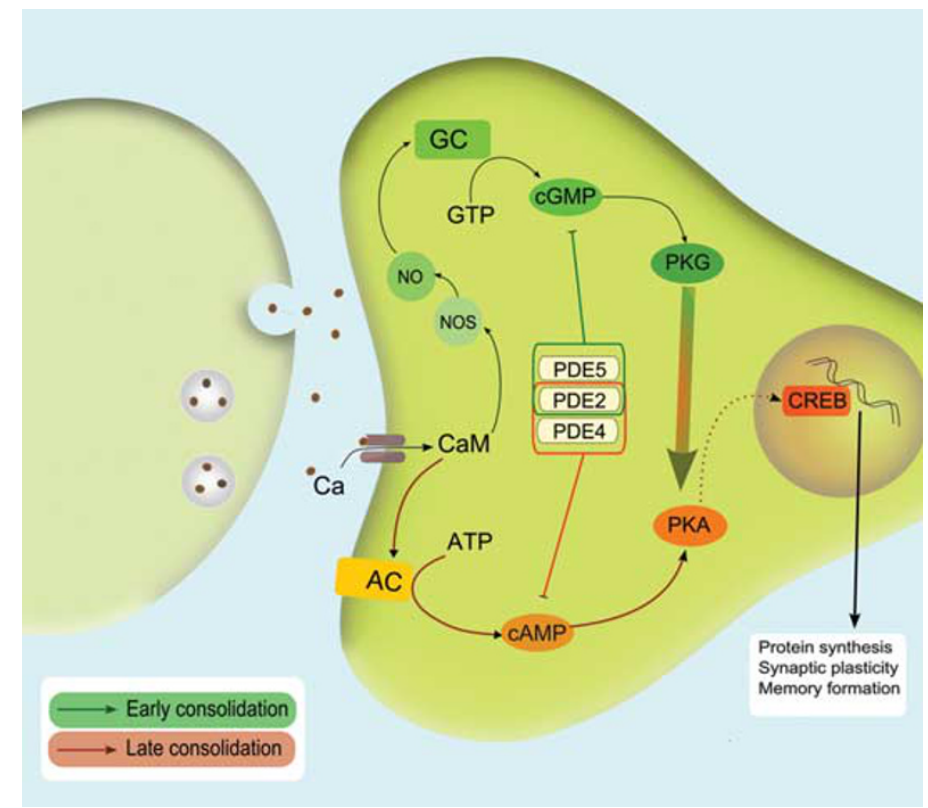

Figure 6 Hypothetical scheme of cGMP-and cAMP-mediated pathways in hippocampal memory consolidation processes. Calcium (Ca $\left.{ }^{2+}\right)$ influx through NMDA receptors activates calmodulin (CaM), which in an early phase mediates the activation of nitric oxide synthase and subsequent nitric oxide (NO). The latter binds to soluble guanylate cyclase (GC), which upon binding stimulates the conversion of guanosine triphosphate (GTP) to cyclic guanosine monophosphate (cGMP). cGMP then hydrolyzes protein kinase G (PKG). As a consequence of a persistent intracellular presence of $\mathrm{Ca}^{2+}$, adenylyl cyclase (AC) will eventually be triggered to convert adenosine triphosphate (ATP) into cyclic adenosine monophosphate. Consequently, cAMP will stimulate protein kinase A (PKA) presumably to enhance CAMP responsive element-binding protein (CREB)-mediated transcription and in that manner promote protein synthesis. Our data show that upregulation of the CGMP-PKG pathway in an early consolidation phase requires CAMP-mediated signaling in a later phase of consolidation. How this mechanistically takes place is not clear. It could be argued that cGMP and/or PKG lower the stimulation threshold for Ca ${ }^{2+}$ signals from other sources then through influx via the NMDA receptor, and therefore could enhance postsynaptic cAMP signaling.

In the present study, we demonstrated that cAMPtargeting and cGMP-targeting PDE inhibitors improve memory through hippocampal cAMP-PKA signaling and cGMP-PKG signaling, respectively. These signaling cascades take part in memory consolidation during two temporally separated phases after learning. cGMP-PKG signaling mediates early consolidation, whereas late consolidation requires activation of the cAMP-PKA cascade. These effects on memory were in line with the LTP studies, lending support to the notion that synaptic changes underlie the nootropic effects of PDE inhibitors. Importantly, we demonstrate for the first time that the early cGMP pathway is unable to enhance consolidation or synaptic plasticity in the absence of late CAMP signaling, implying that cAMP signaling is the common pathway in long-term memory formation.

\section{FUNDING AND DISCLOSURE}

Dr J Prickaerts has received research funds from Intracellular Therapies, Takeda, CoMentis, SK Biopharmaceuticals, Algiax Pharmaceuticals, and Envivo Pharmaceuticals. Dr K. Rutten is a full-time employee of Grünenthal $\mathrm{GmbH}$. The remaining authors declare no conflict of interest.

\section{REFERENCES}

Akkerman S, Blokland A, Reneerkens O, van Goethem NP, Bollen E, Gijselaers HJ et al (2012). Object recognition testing: methodo- logical considerations on exploration and discrimination measures. Behav Brain Res 232: 335-347.

Bach ME, Barad M, Son H, Zhuo M, Lu YF, Shih R et al (1999). Age-related defects in spatial memory are correlated with defects in the late phase of hippocampal long-term potentiation in vitro and are attenuated by drugs that enhance the cAMP signaling pathway. Proc Natl Acad Sci USA 96: 5280-5285.

Barad M, Bourtchouladze R, Winder DG, Golan H, Kandel E (1998). Rolipram, a type IV-specific phosphodiesterase inhibitor, facilitates the establishment of long-lasting long-term potentiation and improves memory. Proc Natl Acad Sci USA 95: 15020-15025.

Bernabeu R, Schmitz P, Faillace MP, Izquierdo I, Medina JH (1996). Hippocampal cGMP and cAMP are differentially involved in memory processing of inhibitory avoidance learning. Neuroreport 7: 585-588.

Blokland A, Schreiber R, Prickaerts J (2006). Improving memory: a role for phosphodiesterases. Curr Pharm Des 12: 2511-2523.

Boess FG, Hendrix M, van der Staay FJ, Erb C, Schreiber R, van Staveren W et al (2004). Inhibition of phosphodiesterase 2 increases neuronal cGMP, synaptic plasticity and memory performance. Neuropharmacology 47: 1081-1092.

Bourtchouladze R, Abel T, Berman N, Gordon R, Lapidus K, Kandel ER (1998). Different training procedures recruit either one or two critical periods for contextual memory consolidation, each of which requires protein synthesis and PKA. Learn Mem 5: 365-374.

Chapman PF, White GL, Jones MW, Cooper-Blacketer D, Marshall VJ, Irizarry $\mathrm{M}$ et al (1999). Impaired synaptic plasticity and learning in aged amyloid precursor protein transgenic mice. Nat Neurosci 2: 271-276.

Devan BD, Pistell PJ, Daffin Jr LW, Nelson CM, Duffy KB, Bowker JL et al (2007). Sildenafil citrate attenuates a complex 
maze impairment induced by intracerebroventricular infusion of the NOS inhibitor N(omega)-nitro-L-arginine methyl ester. Eur J Pharmacol 563: 134-140.

Ennaceur A, Delacour J (1988). A new one-trial test for neurobiological studies of memory in rats. 1: Behavioral data. Behav Brain Res 31: 47-59.

Frey U, Huang YY, Kandel ER (1993). Effects of cAMP simulate a late stage of LTP in hippocampal CA1 neurons. Science 260: 1661-1664.

Izquierdo I, Bevilaqua LR, Rossato JI, Bonini JS, Medina JH, Cammarota M (2006). Different molecular cascades in different sites of the brain control memory consolidation. Trends Neurosci 29: 496-505.

Izquierdo I, Medina JH (1997). Memory formation: the sequence of biochemical events in the hippocampus and its connection to activity in other brain structures. Neurobiol Learn Mem 68: 285-316.

Kandel ER (2001). The molecular biology of memory storage: a dialogue between genes and synapses. Science 294: 1030-1038.

Krause W, Kuhne G (1988). Pharmacokinetics of rolipram in the rhesus and cynomolgus monkeys, the rat and the rabbit. Studies on species differences. Xenobiotica 18: 561-571.

Kroker KS, Rast G, Giovannini R, Marti A, Dorner-Ciossek C, Rosenbrock H (2012). Inhibition of acetylcholinesterase and phosphodiesterase-9A has differential effects on hippocampal early and late LTP. Neuropharmacology 62: 1964-1974.

Lu YF, Hawkins RD (2002). Ryanodine receptors contribute to cGMP-induced late-phase LTP and CREB phosphorylation in the hippocampus. J Neurophysiol 88: 1270-1278.

Lu YF, Kandel ER, Hawkins RD (1999). Nitric oxide signaling contributes to late-phase LTP and CREB phosphorylation in the hippocampus. J Neurosci 19: 10250-10261.

Matsumoto Y, Unoki S, Aonuma H, Mizunami M (2006). Critical role of nitric oxide-cGMP cascade in the formation of cAMPdependent long-term memory. Learn Mem 13: 35-44.

Monti B, Berteotti C, Contestabile A (2006). Subchronic Rolipram Delivery Activates Hippocampal CREB and Arc, Enhances Retention and Slows Down Extinction of Conditioned Fear. Neuropsychopharmacology 31: 278-286.

Navakkode S, Sajikumar S, Frey JU (2004). The type IV-specific phosphodiesterase inhibitor rolipram and its effect on hippocampal long-term potentiation and synaptic tagging. J Neurosci 24: 7740-7744.

Palmeri A, Privitera L, Giunta S, Loreto C, Puzzo D (2013). Inhibition of phosphodiesterase-5 rescues age-related impairment of synaptic plasticity and memory. Behav Brain Res 240: 11-20.

Paterno R, Faraci FM, Heistad DD (1996). Role of Ca(2+)dependent $\mathrm{K}+$ channels in cerebral vasodilatation induced by increases in cyclic GMP and cyclic AMP in the rat. Stroke 27: 1603-1607; discussion 1607-1608.

Paxinos G, Watson C (1998). The Rat Brain in Stereotaxic Coordinates, 4th edn. Academic Press: London.

Prickaerts J, de Vente J, Honig W, Steinbusch HW, Blokland A (2002a). cGMP, but not cAMP, in rat hippocampus is involved in early stages of object memory consolidation. Eur J Pharmacol 436: 83-87.

Prickaerts J, Sik A, van Staveren WC, Koopmans G, Steinbusch HW, van der Staay FJ et al (2004). Phosphodiesterase type 5 inhibition improves early memory consolidation of object information. Neurochem Int 45: 915-928.

Prickaerts J, Steinbusch HW, Smits JF, de Vente J (1997). Possible role of nitric oxide-cyclic GMP pathway in object recognition memory: effects of 7-nitroindazole and zaprinast. Eur $J$ Pharmacol 337: 125-136.
Prickaerts J, van Staveren WC, Sik A, Markerink-van Ittersum M, Niewohner U, van der Staay FJ et al (2002b). Effects of two selective phosphodiesterase type 5 inhibitors, sildenafil and vardenafil, on object recognition memory and hippocampal cyclic GMP levels in the rat. Neuroscience 113: 351-361.

Puzzo D, Staniszewski A, Deng SX, Privitera L, Leznik E, Liu S et al (2009). Phosphodiesterase 5 inhibition improves synaptic function, memory, and amyloid-beta load in an Alzheimer's disease mouse model. J Neurosci 29: 8075-8086.

Reneerkens O, Rutten K, Blokland A, Prickaerts J (2009). Selective phosphodiesterase inhibitors: a promising target for cognition enhancement. Psychopharmacology 202: 419-443.

Reneerkens OA, Rutten K, Akkerman S, Blokland A, Shaffer CL, Menniti FS et al (2012). Phosphodiesterase type 5 (PDE5) inhibition improves object recognition memory: indications for central and peripheral mechanisms. Neurobiol Learn Mem 97: 370-379.

Reneerkens OA, Rutten K, Bollen E, Hage T, Blokland A, Steinbusch HW et al (2013). Inhibition of phoshodiesterase type 2 or type 10 reverses object memory deficits induced by scopolamine or MK-801. Behav Brain Res 236: 16-22.

Rose GM, Hopper A, De Vivo M, Tehim A (2005). Phosphodiesterase inhibitors for cognitive enhancement. Curr Pharm Design 11: 3329-3334.

Rutten K, Lieben C, Smits L, Blokland A (2007a). The PDE4 inhibitor rolipram reverses object memory impairment induced by acute tryptophan depletion in the rat. Psychopharmacology (Berl) 192: 275-282.

Rutten K, Prickaerts J, Blokland A (2006). Rolipram reverses scopolamine-induced and time-dependent memory deficits in object recognition by different mechanisms of action. Neurobiol Learn Mem 85: 132-138.

Rutten K, Prickaerts J, Hendrix M, van der Staay FJ, Sik A, Blokland A (2007b). Time-dependent involvement of cAMP and cGMP in consolidation of object memory: studies using selective phosphodiesterase type 2, 4 and 5 inhibitors. Eur J Pharmacol 558: $107-112$.

Rutten K, Van Donkelaar EL, Ferrington L, Blokland A, Bollen E, Steinbusch HW et al (2009). Phosphodiesterase inhibitors enhance object memory independent of cerebral blood flow and glucose utilization in rats. Neuropsychopharmacology 34: 1914-1925.

Rutten K, Vente JD, Sik A, Ittersum MM, Prickaerts J, Blokland A (2005). The selective PDE5 inhibitor, sildenafil, improves object memory in Swiss mice and increases cGMP levels in hippocampal slices. Behav Brain Res 164: 11-16.

Sakamoto K, Karelina K, Obrietan K (2011). CREB: a multifaceted regulator of neuronal plasticity and protection. J Neurochem 116: $1-9$.

Son H, Lu YF, Zhuo M, Arancio O, Kandel ER, Hawkins RD (1998). The specific role of cGMP in hippocampal LTP. Learn Mem 5: 231-245.

Taqatqeh F, Mergia E, Neitz A, Eysel UT, Koesling D, Mittmann T (2009). More than a retrograde messenger: nitric oxide needs two cGMP pathways to induce hippocampal long-term potentiation. J Neurosci 29: 9344-9350.

Zakharenko SS, Patterson SL, Dragatsis I, Zeitlin SO, Siegelbaum SA, Kandel ER et al (2003). Presynaptic BDNF required for a presynaptic but not postsynaptic component of LTP at hippocampal CA1-CA3 synapses. Neuron 39: 975-990.

Zhang HT, Huang Y, Suvarna NU, Deng C, Crissman AM, Hopper AT et al (2005). Effects of the novel PDE4 inhibitors MEM1018 and MEM1091 on memory in the radial-arm maze and inhibitory avoidance tests in rats. Psychopharmacology (Berl) 179: 613-619.

Supplementary Information accompanies the paper on the Neuropsychopharmacology website (http://www.nature.com/npp) 